\title{
Sleep restriction increases free fatty acids in healthy men
}

\author{
Josiane L. Broussard • Florian Chapotot • \\ Varghese Abraham • Andrew Day • Fanny Delebecque • \\ Harry R. Whitmore • Esra Tasali
}

Received: 29 November 2014 / Accepted: 22 December 2014 /Published online: 22 February 2015

(C) Springer-Verlag Berlin Heidelberg 2015

\begin{abstract}
Aims/hypothesis Sleep loss is associated with insulin resistance and an increased risk for type 2 diabetes, yet underlying mechanisms are not understood. Elevation of circulating nonesterified (i.e. free) fatty acid (NEFA) concentrations can lead to insulin resistance and plays a central role in the development of metabolic diseases. Circulating NEFA in healthy individuals shows a marked diurnal variation with maximum levels occurring at night, yet the impact of sleep loss on NEFA levels across the $24 \mathrm{~h}$ cycle remains unknown. We hypothesised that sleep restriction would alter hormones that are known to stimulate lipolysis and lead to an increase in NEFA levels.

Methods We studied 19 healthy young men under controlled laboratory conditions with four consecutive nights of $8.5 \mathrm{~h}$ in bed (normal sleep) and $4.5 \mathrm{~h}$ in bed (sleep restriction) in randomised order. The $24 \mathrm{~h}$ blood profiles of NEFA, growth hormone $(\mathrm{GH})$, noradrenaline (norepinephrine), cortisol, glucose and insulin were simultaneously assessed. Insulin sensitivity was estimated by a frequently sampled intravenous glucose tolerance test.

Results Sleep restriction relative to normal sleep resulted in increased NEFA levels during the nocturnal and earlymorning hours. The elevation in NEFA was related to
\end{abstract}

J. L. Broussard ( $\square)$

Diabetes and Obesity Research Institute, Cedars-Sinai Medical

Center, 8700 Beverly Blvd, THA E104, Los Angeles,

CA 90048, USA

e-mail: josiane@uchicago.edu

F. Chapotot $\cdot$ V. Abraham $\cdot$ F. Delebecque $\cdot$ H. R. Whitmore $\cdot$

E. Tasali

Department of Medicine, University of Chicago, Chicago, IL, USA

A. Day

Department of Medicine, University of Wisconsin Hospitals and

Clinics, Madison, WI, USA prolonged nocturnal GH secretion and higher early-morning noradrenaline levels. Insulin sensitivity was decreased after sleep restriction and the reduction in insulin sensitivity was correlated with the increase in nocturnal NEFA levels.

Conclusions/interpretation Sleep restriction in healthy men results in increased nocturnal and early-morning NEFA levels, which may partly contribute to insulin resistance and the elevated diabetes risk associated with sleep loss.

Keywords Catecholamines - Cortisol · Free fatty acids . Glucose · Growth hormone - Insulin sensitivity · Lipolysis . Metabolism · NEFA - Sleep loss · Sleep restriction · Type 2 diabetes
Abbreviations
GH Growth hormone
REM Rapid eye movement
NEFA Non-esterified fatty acid
IVGTT Intravenous glucose tolerance test

\section{Introduction}

Substantial evidence from population studies suggests that individuals who regularly curtail their sleep are at an increased risk for developing type 2 diabetes [1]. Several well-controlled laboratory studies have consistently shown marked reductions in insulin sensitivity in young adults following sleep loss [2-7]. However, the mechanisms by which insufficient sleep increases type 2 diabetes risk are not known. Alterations in non-esterified (i.e. free) fatty acid (NEFA) levels play a central role in the pathogenesis of insulin resistance and the development of metabolic diseases [8, 9]. Acute elevation in circulating NEFA levels in healthy individuals has been linked to insulin resistance in multiple investigations [10-13]. A marked diurnal variation in circulating NEFA concentrations 
has been observed in healthy individuals with peak levels occurring in the middle of the night and declining levels towards morning [14]. To date, the impact of sleep loss on NEFA concentrations across the $24 \mathrm{~h}$ cycle remains unknown.

Growth hormone (GH), noradrenaline (norepinephrine) and cortisol are major signals that link the central nervous system to peripheral tissues. In addition, these hormones are known to increase the rate of lipolysis, resulting in elevated circulating NEFA concentrations. Experimental sleep restriction in healthy young adults has been associated with alterations in nocturnal GH secretion [15], increased night-time noradrenaline concentrations [5] and elevated evening cortisol levels $[4,16,17]$. We therefore hypothesised that sleep loss would alter these lipolytic hormones, leading to an increase in NEFA concentrations. To test this hypothesis, we simultaneously assessed $24 \mathrm{~h}$ blood profiles of NEFA, GH, noradrenaline and cortisol in healthy volunteers who were tested in the laboratory under conditions of normal sleep and sleep restriction. We also assessed $24 \mathrm{~h}$ glucose and insulin profiles and estimated insulin sensitivity by an intravenous glucose tolerance test during normal sleep and sleep restriction.

\section{Methods}

Participants Nineteen healthy young lean men between 18 and 30 years of age were recruited from the community through local advertisements. Exclusion criteria were a history of any chronic medical condition, acute illness, shift work, travel across time zones during the past 4 weeks, depressed mood (as assessed by a score on the Center for Epidemiologic Studies of Depression Scale $>16$ ), use of any prescription or over-the-counter medications or supplements known to affect sleep or glucose metabolism, current smoking, substantial consumption of alcohol ( $>2$ drinks per day) or caffeine (>300 mg per day) or abnormal findings on physical examination or routine laboratory testing. All participants had an overnight laboratory polysomnography to exclude sleep disorders, as well as a standard $75 \mathrm{~g}$ oral glucose tolerance test and fasting blood sample collection for routine laboratory tests, including complete blood counts, a comprehensive metabolic panel, thyroid function tests, a lipid panel and $\mathrm{HbA}_{1 \mathrm{c}}$ measurement. A 12-lead electrocardiogram was also obtained to screen for sleep abnormalities. Healthy participants who had normal glucose tolerance and no current or previous sleep disorders were included. All participants had regular selfreported nocturnal time in bed of 7.5-8.5 h. The Institutional Review Board of the University of Chicago approved the protocol and all participants gave written informed consent.

Experimental protocol Participants were studied at the University of Chicago Clinical Resource Center under two experimental conditions in randomised order spaced at least
4 weeks apart: (1) four consecutive nights of $8.5 \mathrm{~h}$ in bed (normal sleep) and (2) four consecutive nights of $4.5 \mathrm{~h}$ in bed (sleep restriction). Sleep was recorded by polysomnography (Nihon Kohden, Irvine, CA, USA) during each night in each sleep condition. The recordings were visually scored in $30 \mathrm{~s}$ epochs as rapid eye movement (REM) sleep, non-REM sleep and wake according to standard criteria [18]. Participants remained in the laboratory during the entire protocol and participated in sedentary activities (e.g. reading, watching TV, computer work, board or card games, etc.). A research staff member was continuously present to monitor wakefulness. During the week preceding each inpatient period, participants maintained standardised bedtimes at home in accordance with their usual habits. Naps were not allowed. Compliance with this schedule was verified by sleep diaries and continuous wrist activity monitoring (Actiwatch; PhilipsRespironics, Bend, OR, USA).

Blood samples were collected at 15-30 min intervals for $24 \mathrm{~h}$ (from 21:30 hours starting on the evening prior to the third night of each condition) for measurements of NEFA, $\mathrm{GH}$, noradrenaline, cortisol, glucose and insulin (Fig. 1). During waking hours, blood samples were collected while participants remained semi-recumbent at a $30^{\circ}$ angle. During sleep hours, the intravenous line was extended and fed through a port in the wall, allowing for blood drawing from an adjacent room. Diet was strictly controlled (2 day cycle menu of three isoenergetic meals per day) and energy intake was identical under both sleep conditions. Percentage body fat was assessed during each condition by bioimpedance. Participants were not allowed to consume any food or beverages that were not provided by the metabolic kitchen. No caffeine-containing beverages were allowed. Participants ate identical carbohydrate-rich (65\%) meals at 09:00, 14:00 and 19:00 hours during the blood-sampling period. Each meal was completely consumed within $20 \mathrm{~min}$. At 10:00 hours on the day following the fourth night of each sleep condition, a frequently sampled intravenous glucose tolerance test (IVGTT) was performed as previously described [6]. In six IVGTTs during the normal sleep condition and two IVGTTs during the sleep restriction condition, technical difficulties invalidated the estimation of insulin sensitivity and secretion. Five of the six invalid IVGTTs during the normal sleep condition were repeated on a separate day after one night of laboratory polysomnography under the same sleep and experimental conditions. We previously reported the effects of sleep restriction on total body insulin sensitivity and insulin signalling in adipocytes from six men who were included in this report [6].

Hormonal measurements All hormonal values were interpolated at $15 \mathrm{~min}$ intervals to facilitate chronobiological analyses. The NEFA profiles were smoothed using a two-point moving average to facilitate the identification of the onset 
Normal sleep 23:00 - 07:30

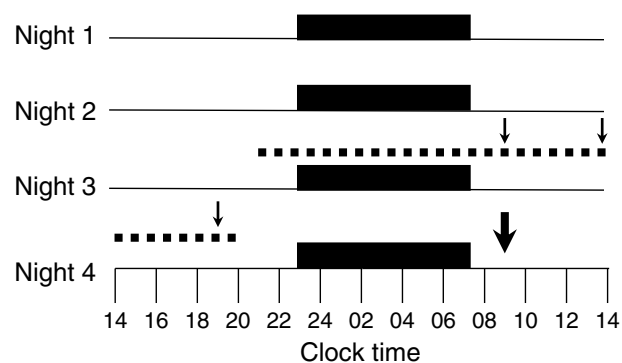

Fig. 1 Study protocol. Black bars, consecutive nights with bedtimes from 23:00 hours to 07:30 hours in the normal sleep condition; red bars, consecutive nights with bedtimes from 01:00 hours to 05:30 hours in the

and peak nocturnal NEFA levels. To better assess the relationships between the GH secretion and NEFA rise, the nocturnal NEFA onset was defined as the minimum NEFA value occurring after the onset of the first GH pulse. The nocturnal NEFA peak was defined as the maximum NEFA value that occurred after the sleep-onset GH pulse. The duration of nocturnal NEFA rise was calculated from NEFA onset to peak. Significant GH secretory pulses were identified as previously described [19]. The duration of nocturnal GH secretion was defined as the total duration of significant GH secretory pulses (concentration above $0.3 \mathrm{ng} / \mathrm{ml}$ ) from the onset of the first $\mathrm{GH}$ pulse after blood sampling began until the end of the sleeponset GH pulse.

Blood samples were centrifuged immediately at $4^{\circ} \mathrm{C}$ and plasma was frozen and stored at $-80^{\circ} \mathrm{C}$ until assay. For each $24 \mathrm{~h}$ profile, all samples obtained from the same individual were measured in the same assay. Plasma NEFA levels were measured using a colorimetric assay (Wako Chemicals, Richmond, VA, USA). Plasma glucose was assayed by the glucose oxidase method with a coefficient of variation $<2 \%$. Serum insulin and plasma cortisol and GH were measured by chemiluminescence assays using the Immulite Immunochemistry System (Diagnostic Products Corporation, Los Angeles, CA, USA). Plasma noradrenaline was measured using an HPLC system (Coulochem MD5001; ESA, Chelmsford, MA, USA) with a lower limit of sensitivity of $10 \mathrm{pg} / \mathrm{ml}$ and an intra-assay coefficient of variation of 6$7.8 \%$.

Statistical analyses The effects of sleep restriction on metabolic variables were assessed using repeated measures design by mixed-model ANOVA with participants as random factor and sleep conditions (normal sleep, sleep restriction) as fixed factors. The NEFA time points between normal sleep and sleep restriction conditions were analysed by mixed-model ANOVA with participants as random factor and sleep condition and sampling time as fixed factors after controlling for multiple comparisons with a Benjamini and Hochberg procedure. Changes in hormonal and metabolic variables following
Sleep restriction 01:00 - 05:30

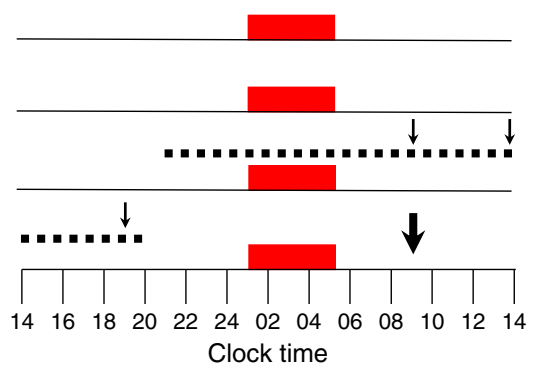

sleep restriction condition; dotted lines, $24 \mathrm{~h}$ blood-sampling period; thin arrows, identical meals served at 09:00, 14:00 and 19:00 hours; thick arrows, IVGTT

sleep restriction were expressed as percentage of the values obtained during normal sleep. Relationships between changes in NEFA levels and changes in other metabolic variables were examined using the Pearson coefficient. Data are reported as mean and SEM. All statistical analyses were performed using JMP software (version 9.0.3; SAS Institute, Cary, NC, USA). A $p$ value $<0.05$ was considered significant for all analyses.

\section{Results}

Nineteen men (mean age: $23.5 \pm 0.7$ years) participated in the study. Average body mass index $(23.4 \pm 0.4$ vs $23.3 \pm 0.4$ $\left.\mathrm{kg} / \mathrm{m}^{2} ; p=0.12\right)$ and percentage body fat $(19.3 \pm 1.1 \% \mathrm{vs}$ $19.2 \pm 1.0 \% ; p=0.86$ ) did not differ between sleep conditions. On average, participants slept for $7.8 \pm 0.1 \mathrm{~h}$ during normal sleep vs $4.3 \pm 0.0 \mathrm{~h}$ during sleep restriction (Table 1 ).

The $24 \mathrm{~h}$ profile of NEFA displayed a marked circadian variation under both sleep conditions (Fig. 2a). When sleep was restricted, the duration of the nocturnal NEFA increase was prolonged by approximately $50 \mathrm{~min}$ (Table 2 ). Sleep restriction resulted in a plateau of NEFA levels in the morning rather than the continued decline observed during normal sleep. Sleep restriction relative to normal sleep resulted in a significant increase in mean NEFA levels by approximately 15-30\% during the nocturnal and early-morning hours (Fig. 2a, Table 2). We also analysed NEFA AUCs for the same time periods and found similar results.

During normal sleep, a single nocturnal GH pulse was observed in all but four of the 19 participants (Fig. 2b). In contrast, during sleep restriction 14 of the 19 participants showed a biphasic nocturnal GH release, resulting in GH secretion being prolonged by approximately $50 \mathrm{~min}$. The total amount of nocturnal GH secreted did not differ between sleep conditions (Table 2). The increase in the duration of nocturnal GH secretion was correlated with the prolonged duration of NEFA rise $(r=0.81, p<0.0001)$, while no relationship was found with the amount of nocturnal GH $(r=0.30, p=0.207)$. 
Table 1 Effects of sleep restriction on sleep stages

\begin{tabular}{llll}
\hline Sleep variable & Normal sleep & Sleep restriction & $p$ value \\
\hline All nights & & & \\
N1, min & & & $<0.0001$ \\
N1, \% & $20.6 \pm 1.4$ & $6.1 \pm 0.5$ & $<0.0001$ \\
N2, min & $4.5 \pm 0.3$ & $2.4 \pm 0.2$ & $<0.0001$ \\
N2, \% & $248.3 \pm 4.9$ & $118.9 \pm 4.8$ & $<0.0001$ \\
Slow wave sleep, min & $53.1 \pm 1.0$ & $45.8 \pm 1.8$ & 0.13 \\
Slow wave sleep, \% & $15.9 \pm 1.3$ & $30.0 \pm 2.1$ & $<0.0001$ \\
REM, min & $124.8 \pm 4.5$ & $57.0 \pm 2.6$ & $<0.0001$ \\
REM, \% & $26.6 \pm 0.86$ & $21.9 \pm 1.0$ & $<0.0001$ \\
Night 3 & & & \\
N1, min & $26.1 \pm 2.3$ & $7.0 \pm 1.3$ & $<0.0001$ \\
N1, \% & $5.7 \pm 0.6$ & $2.8 \pm 0.6$ & 0.002 \\
N2, min & $247.1 \pm 5.0$ & $115.6 \pm 6.9$ & $<0.0001$ \\
N2, \% & $53.8 \pm 1.4$ & $44.8 \pm 2.5$ & 0.0005 \\
Slow wave sleep, min & $68.9 \pm 6.8$ & $72.1 \pm 6.4$ & 0.17 \\
Slow wave sleep, \% & $14.9 \pm 1.4$ & $28.1 \pm 2.5$ & $<0.0001$ \\
REM, min & $118.5 \pm 6.8$ & $62.7 \pm 3.7$ & $<0.0001$ \\
REM, \% & $25.6 \pm 1.3$ & $24.3 \pm 1.4$ & 0.50 \\
\hline
\end{tabular}

Data represent mean \pm SEM $(N=19)$. Percentages for each sleep stage were calculated as the percentage of total sleep time

${ }^{a}$ Data are from the average of four nights during each sleep condition

${ }^{\mathrm{b}}$ Data are from night 3 only (i.e. $24 \mathrm{~h}$ blood-sampling night)

$\mathrm{N} 1$, stage 1 non-REM sleep; N2, stage 2 non-REM sleep

Noradrenaline levels were increased by approximately $30 \%$ during the early-night and early-morning hours, when participants had to remain awake during sleep restriction (Fig. 2c, Table 2). This early-night increase in noradrenaline did not appear to influence the NEFA peak or the slope of NEFA rise. However, the early-morning increase in noradrenaline was positively correlated with the early-morning increase in NEFA levels $(r=0.55, p=0.015)$. The normal postawakening cortisol peak occurred approximately $2 \mathrm{~h}$ earlier when sleep was restricted (Fig. 2d) and cortisol levels were $23 \%$ higher in the evening hours as well as during the early night with extended wakefulness (Table 2). The alterations in cortisol secretory patterns did not correlate with any changes in NEFA ( $p>0.05$ for all variables).

Overnight (21:30-09:00 hours) mean fasting insulin levels were higher after sleep restriction as compared with normal sleep $(51.0 \pm 5.1 \mathrm{vs} 46.7 \pm 5.1 \mathrm{pmol} / 1 ; p=0.036)$ despite similar glucose levels $(5.08 \pm 0.07$ vs $5.05 \pm 0.07 \mathrm{mmol} / 1 ; p=0.410)$, suggesting an insulin-resistant state (Fig. 3). When sleep was restricted, the AUC for glucose response to breakfast tended to be higher $(p=0.061)$ and the AUC for insulin response was significantly increased ( $p=0.045$ ), suggesting decreased insulin sensitivity (Fig. 4). The post-breakfast change in insulin level was positively correlated with the change in nocturnal NEFA level $(r=0.51, p=0.025)$.
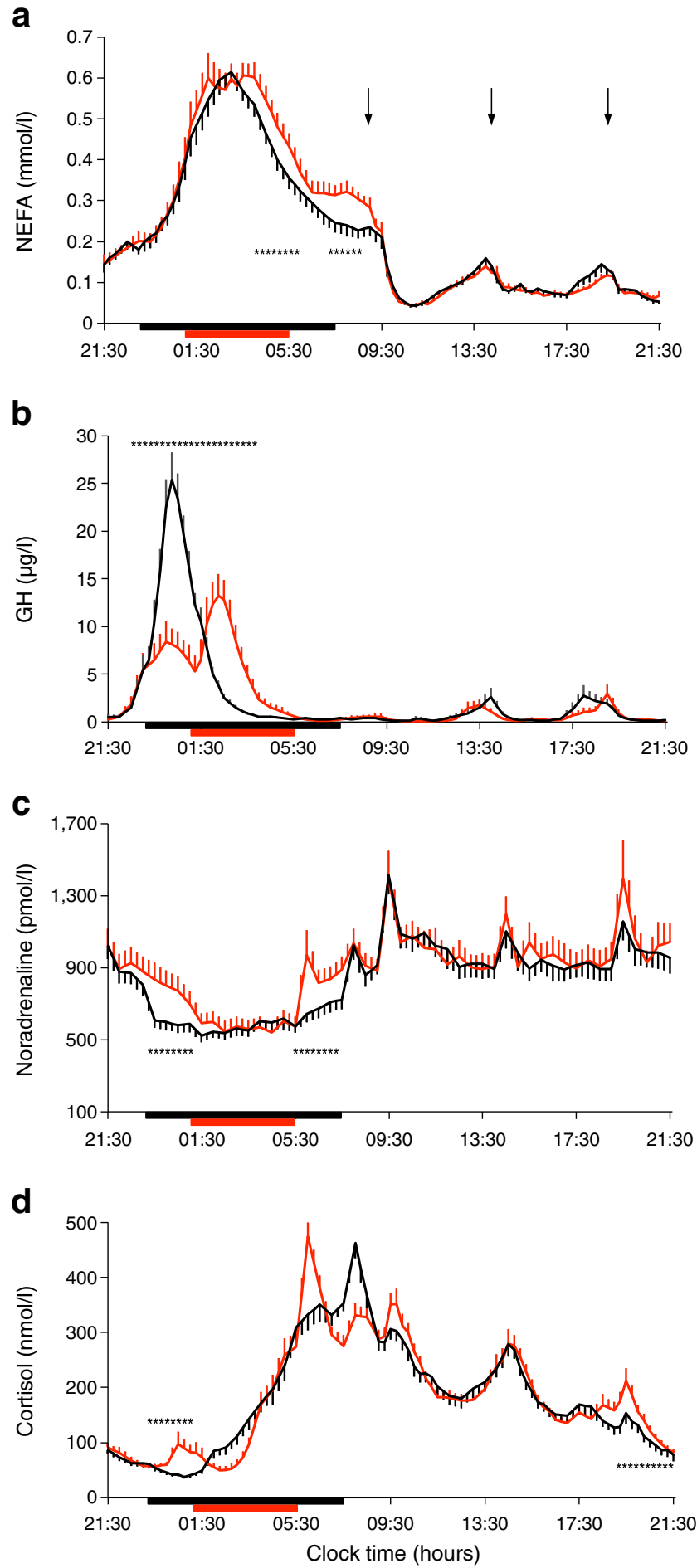

Fig. 2 Twenty-four hour profiles of NEFA (a), GH (b), noradrenaline (c) and cortisol (d) under conditions of normal sleep (black lines) and sleep restriction (red lines) in $N=19$ participants. Error bars are SEM. Horizontal black bars, time in bed under normal sleep (23:00-07:30 hours); horizontal red bars, time in bed under sleep restriction (01:00-05:30 hours); arrows, identical meals served at 09:00, 14:00 and 19:00 hours. ${ }^{*} p<0.05$ for sleep restriction vs normal sleep at specific time points

Insulin sensitivity estimated by the IVGTT was reduced by $23 \%$ during sleep restriction as compared with normal sleep 
Table 2 Effects of sleep restriction on $24 \mathrm{~h}$ profiles of NEFA, $\mathrm{GH}$, noradrenaline and cortisol

Data represent mean \pm SEM hormone levels during the time interval indicated within parentheses $(N=19)$

\begin{tabular}{llll}
\hline Variable & Normal sleep & Sleep restriction & $p$ value \\
\hline NEFA & & & \\
$\quad$ Nocturnal onset, mmol/1 & $0.15 \pm 0.01$ & $0.15 \pm 0.01$ & 0.820 \\
$\quad$ Nocturnal peak, mmol/1 & $0.66 \pm 0.03$ & $0.69 \pm 0.04$ & 0.436 \\
$\quad$ Duration of nocturnal increase, min & $210 \pm 15$ & $262 \pm 15$ & 0.003 \\
$\quad$ Mean nocturnal (04:00-05:45 hours), mmol/1 & $0.44 \pm 0.02$ & $0.51 \pm 0.03$ & 0.014 \\
Mean early morning (07:15-09:00 hours), mmol/1 & $0.24 \pm 0.02$ & $0.31 \pm 0.02$ & 0.019 \\
$\quad$ Mean daytime (09:00-21:30 hours), mmol/1 & $0.10 \pm 0.01$ & $0.09 \pm 0.01$ & 0.565 \\
GH & & & 0.004 \\
$\quad$ Duration of nocturnal secretion, min & $307 \pm 18$ & $359 \pm 20$ & 0.156 \\
Nocturnal amount secreted, $\mu \mathrm{g}$ & $726 \pm 59$ & $614 \pm 57$ & 0.336 \\
$\quad$ Daytime amount secreted, $\mu \mathrm{g}$ & $127 \pm 21$ & $103 \pm 17$ & \\
Noradrenaline & & & 0.002 \\
$\quad$ Mean early night (23:00-01:00 hours), pmol/1 & $633 \pm 52$ & $833 \pm 94$ & 0.038 \\
$\quad$ Mean early morning (05:30-07:30 hours), pmol/1 & $676 \pm 50$ & $868 \pm 90$ & 0.781 \\
$\quad$ Mean daytime (09:00-21:30 hours), pmol/1 & $1,064 \pm 74$ & $1,085 \pm 98$ & \\
Cortisol & & & 0.008 \\
$\quad$ Mean early night (23:00-01:00 hours), nmol/1 & $51 \pm 5$ & $75 \pm 9$ & 0.047 \\
Mean evening (19:00-21:30 hours), nmol/1 & $119 \pm 12$ & $146 \pm 12$ & \\
\hline
\end{tabular}

( $p=0.004$, Fig. 5a). The acute insulin response to glucose did not differ between sleep conditions $(p=0.22)$. Thus, the lack of compensatory insulin secretion after sleep restriction resulted in a lower disposition index $(p=0.042)$, indicating an increase in diabetes risk. The decrease in insulin sensitivity was correlated with the increase in mean nocturnal NEFA levels $(r=-0.52 ; p=0.05 ;$ Fig. $5 \mathrm{~b})$ and the AUC for nocturnal NEFA levels $(r=-0.53, p=0.05)$. The decrease in insulin sensitivity did not correlate with any changes in noradrenaline, cortisol or $\mathrm{GH}$ ( $p>0.10$ for all variables).
Fig. 3 Twenty-four hour profiles of glucose (a, b) and insulin (c, d) under conditions of normal sleep (black lines) and sleep restriction (red lines) in $N=19$ participants. Error bars are SEM. Horizontal black bars, time in bed under normal sleep (23:00

07:30 hours); horizontal red bars, time in bed under sleep restriction (01:00-05:30 hours); arrows, identical meals served at 09:00, 14:00 and 19:00 hours; vertical lines, AUC over the first postprandial $2.5 \mathrm{~h}$ after the breakfast meal
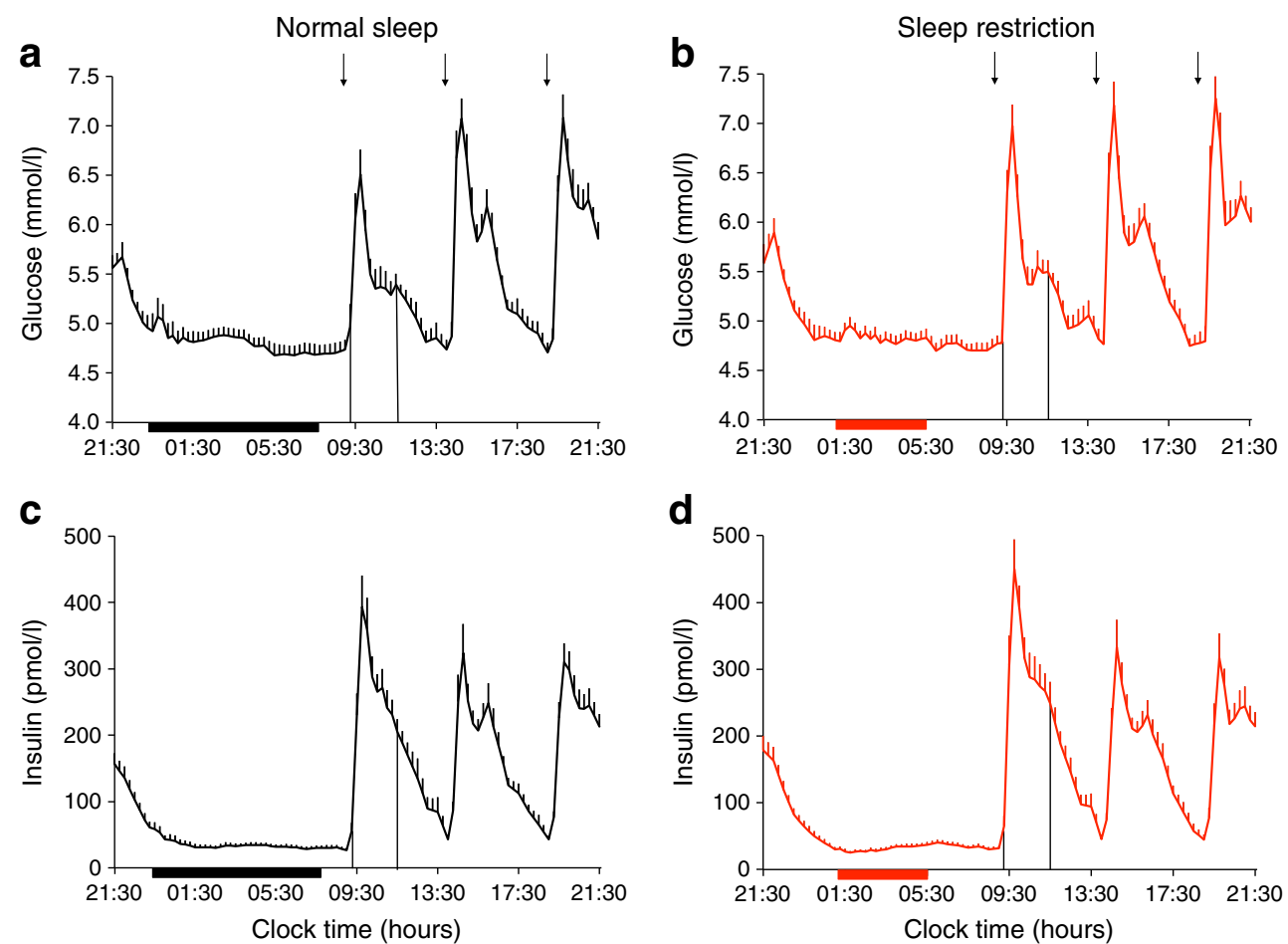


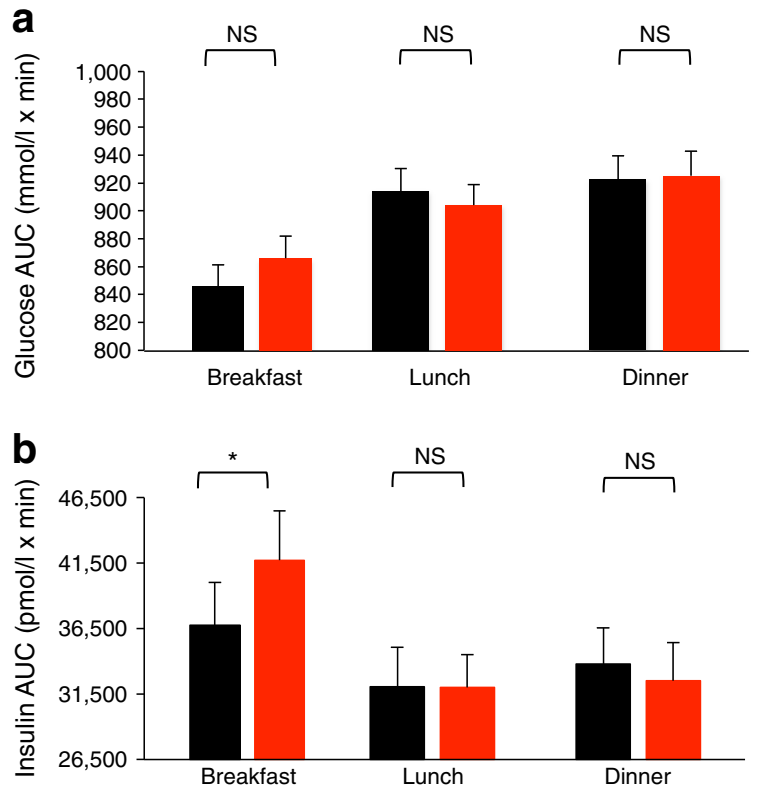

Fig. 4 AUC values for glucose (a) and insulin (b) after breakfast, lunch and dinner under conditions of normal sleep (black bars) and sleep restriction (red bars) in $N=19$ participants. Error bars are SEM. Identical meals were served at 09:00, 14:00 and 19:00 hours. The AUCs were calculated using the trapezoidal method over the postprandial $2.5 \mathrm{~h}$ for each meal. ${ }^{*} p<0.05$ sleep restriction vs normal sleep

\section{Discussion}

We have demonstrated that experimental sleep restriction in healthy young lean men results in an increase in nocturnal and early-morning NEFA levels accompanied by insulin resistance. We also found that alterations in two lipolytic hormones, namely GH and noradrenaline, may be involved in the observed increase in NEFA levels during sleep restriction. These findings provide novel insights into our understanding of the pathways that may link sleep restriction to insulin resistance and increased diabetes risk.

To our knowledge, this is the first study to examine the impact of sleep loss on $24 \mathrm{~h}$ NEFA profiles. We observed the maximum NEFA levels occurring at night, as reported in earlier studies [20-23]. When sleep was restricted, the NEFA levels remained elevated during the nocturnal and earlymorning hours in the prolonged fasted state. However, daytime NEFA levels were similar between sleep conditions, suggesting that sleep restriction may differentially affect NEFA levels during fasting vs fed states. Thus far, two studies have explored the effects of sleep deprivation on NEFA concentrations, but measurements were taken only in the morning over a limited number of time points $[24,25]$. In the first study, NEFA concentrations were measured following one night of total sleep deprivation and recovery sleep in overweight individuals. Morning fasting and postprandial NEFA concentrations were found to be lower after recovery sleep as compared with baseline [25]. In the second study, 2 weeks of sleep restriction during diet-induced weight loss resulted in increased morning fasting NEFA levels in overweight adults [24].

To examine the potential signals that may be involved in elevations in NEFA during sleep restriction, we simultaneously assessed $24 \mathrm{~h}$ profiles of $\mathrm{GH}$, noradrenaline and cortisol (i.e. hormones that are known to stimulate lipolysis and link the central nervous system to peripheral metabolism). We found that the nocturnal GH secretory pattern became biphasic during sleep restriction, relative to normal sleep, resulting in extended GH secretion, as previously reported [15]. The increase in the duration of nocturnal GH secretion was strongly related to the prolonged nocturnal increase in NEFA, suggesting that an altered GH secretory pattern may be partially responsible for the observed increase in nocturnal NEFA levels during sleep restriction. However, it is also possible that the prolonged exposure of peripheral tissues to higher GH levels

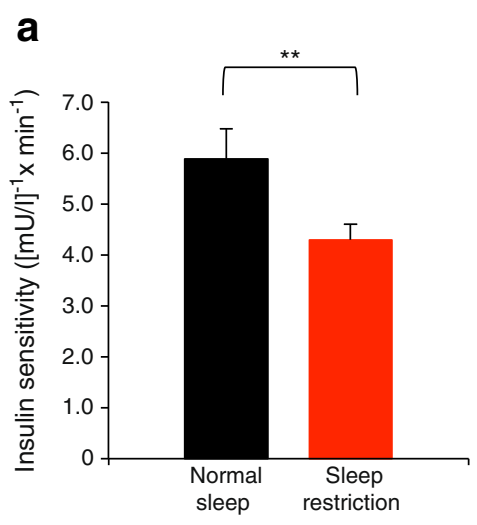

Fig. 5 (a) Insulin sensitivity from IVGTTs under conditions of normal sleep (black bars) and sleep restriction (red bars) in $N=19$ participants. ${ }^{* *} p<0.01$ sleep restriction vs normal sleep. Error bars are SEM. To convert values to SI units, multiply by 0.167 . (b) Correlation between the b

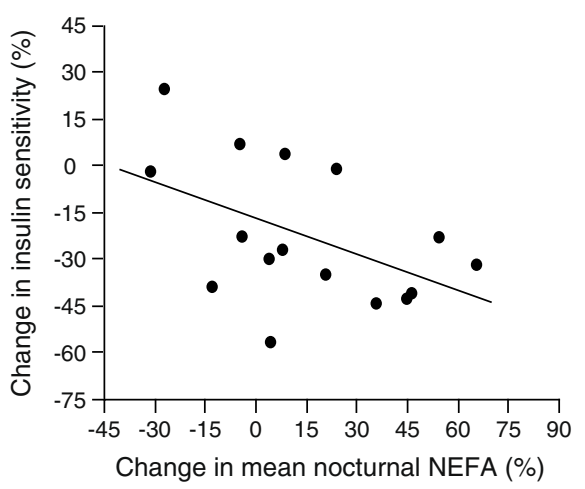

change in nocturnal NEFA levels and the change in insulin sensitivity in $n=16$ participants. Changes during sleep restriction are expressed as percentage of the values obtained during normal sleep. $r=-0.52 ; p=0.05$ 
may directly affect glucose and lipid regulation at the tissues themselves.

We also found that noradrenaline levels were elevated during the early-night and early-morning hours when participants had to remain awake during the sleep restriction condition, consistent with the known inhibitory effect of sleep on noradrenaline [26]. The increase in early-morning noradrenaline was positively correlated with the increase in early-morning NEFA levels, suggesting that higher noradrenaline levels likely contributed to the interruption of decline in morning NEFA concentrations during sleep restriction. Our finding of elevated noradrenaline supports the hypothesis that augmented sympathetic drive may be a potential mediator of the metabolic consequences of sleep restriction and is in agreement with some [5] but not all [24, 27] studies reporting increased sympathetic activity with sleep loss.

Consistent with earlier reports $[4,28]$, we found an increase in evening cortisol levels during sleep restriction. Additionally, we observed an earlier cortisol peak during sleep restriction relative to normal sleep, likely due to the stimulating effects of morning awakening. During the early night, cortisol levels were higher when sleep was restricted, possibly due to the lack of inhibitory effects of the early sleep period. However, we did not find any correlations between changes in cortisol secretion and NEFA levels. Although this may suggest that cortisol is not a key mediator of NEFA release during sleep restriction, we cannot exclude the interactions and combined effects of various hormones, including cortisol, that are affected by sleep loss.

As shown previously in multiple studies [3-6, 29], we observed reduced insulin sensitivity in our participants in the morning hours during sleep restriction as compared with normal sleep. The reduction in morning insulin sensitivity was correlated with the increase in nocturnal NEFA levels. Although this correlation does not demonstrate a direct causal link, acute elevation in circulating NEFA concentrations has been shown to induce insulin resistance in healthy participants in multiple previous studies [10-13]. In addition, the degree of insulin resistance in patients with type 2 diabetes correlates with the elevation in nocturnal NEFA levels [30]. Although the increase in NEFA levels during sleep restriction was modest (an increase of approximately 15-30\% during nocturnal and early-morning hours) in our healthy participants, these findings are consistent with the notion that even a very small sustained increase in NEFA concentrations can alter insulin sensitivity [31]. Our findings are also in agreement with the observation of elevated nocturnal NEFA levels in a dog model of diet-induced insulin resistance [32]. Although we did not find significant correlations between decreased insulin sensitivity and changes in cortisol, noradrenaline or $\mathrm{GH}$, alterations in these factors are known to affect insulin sensitivity and thus may be additional mediators of the insulin-resistant state that we observed in our participants.
Future mechanistic studies (e.g. use of pharmacological agents to suppress NEFA) will be necessary to demonstrate a direct causal role for NEFA in insulin resistance induced by sleep loss. Our current finding of elevated NEFA also suggests that adipocytes are resistant to the antilipolytic effects of insulin, and thus is consistent with our previous report of impaired intracellular insulin signalling in adipose tissue following sleep restriction [6]. It is important to note that our study does not provide information on the long-term effects of sleep restriction or the effects of recovery sleep following short-term sleep restriction on NEFA levels or insulin sensitivity.

We cannot exclude the possibility that altered circadian timing may play a role in our findings. Sleep and circadian rhythms are highly integrated such that sleep loss can occur with circadian misalignment and studies of sleep restriction have shown evidence for altered circadian timing [33, 34]. Disruption of circadian rhythms has also been associated with adverse effects on metabolism [29, 35]. Additionally, recent findings suggest the importance of clock genes in regulating lipolytic activity in adipose tissue [36]. Future studies are required to determine the independent effects of dysregulated circadian timing on NEFA metabolism.

In summary, we have demonstrated that sleep restriction in healthy young men results in elevations in nocturnal and early-morning NEFA levels accompanied by marked alterations in hormones involved in lipolysis and reduced insulin sensitivity. These findings provide evidence for potential mechanisms by which sleep restriction may be associated with insulin resistance and increased type 2 diabetes risk. From a clinical perspective, our findings support the importance of sleep in the regulation of metabolism and provide further evidence that insufficient sleep, a highly prevalent condition in our modern society, may adversely affect fatty acid metabolism. Future studies are required to discover whether achieving adequate sleep can improve metabolic outcomes and thus be a novel strategy to counteract the current epidemics of diabetes and obesity.

Acknowledgements The authors wish to thank E. Van Cauter, Department of Medicine, University of Chicago, for her expertise and intellectual contributions to this manuscript. We thank T. Marcinkowski, Department of Medicine, University of Chicago, for performing the noradrenaline assays, P. Rue, Department of Medicine, University of Chicago, for performing the insulin and GH assays, the nursing and dietary staff of the University of Chicago General Clinical Resource Center for their expert assistance and the volunteers for participating in this study.

Funding This work was supported by NIH grants R01-HL-075079, P01-AG11412, CTSA-UL1 TR000430, P50-HD057796, P60DK20595, Department of Defense award W81XWH-07-2-0071 and Society in Science-The Branco Weiss Fellowship (to JLB).

Duality of interest The authors declare that there is no duality of interest associated with this manuscript. 
Contribution statement JLB contributed to study design, data acquisition and analysis and wrote the manuscript. FC, VA, AD, FD and HRW contributed to data acquisition and analysis, reviewed and edited the manuscript for intellectual content. ET obtained funding, designed the study, collected and analysed data and wrote the manuscript. All authors gave final approval of the version to be published. ET is the guarantor of this work.

\section{References}

1. Cappuccio FP, D'Elia L, Strazzullo P, Miller MA (2010) Quantity and quality of sleep and incidence of type 2 diabetes: a systematic review and meta-analysis. Diabetes Care 33:414-420

2. Spiegel K, Leproult R, van Cauter E (1999) Impact of sleep debt on metabolic and endocrine function. Lancet 354:1435-1439

3. Spiegel K, Knutson K, Leproult R, Tasali E, van Cauter E (2005) Sleep loss: a novel risk factor for insulin resistance and type 2 diabetes. J Appl Physiol 99:2008-2019

4. Buxton OM, Pavlova M, Reid EW, Wang W, Simonson DC, Adler GK (2010) Sleep restriction for 1 week reduces insulin sensitivity in healthy men. Diabetes 59:2126-2133

5. Nedeltcheva AV, Kessler L, Imperial J, Penev PD (2009) Exposure to recurrent sleep restriction in the setting of high caloric intake and physical inactivity results in increased insulin resistance and reduced glucose tolerance. J Clin Endocrinol Metab 94:3242-3250

6. Broussard JL, Ehrmann DA, van Cauter E, Tasali E, Brady MJ (2012) Impaired insulin signaling in human adipocytes after experimental sleep restriction: a randomized, crossover study. Ann Intern Med 157:549-557

7. Donga E, van Dijk M, van Dijk JG et al (2010) A single night of partial sleep deprivation induces insulin resistance in multiple metabolic pathways in healthy participants. J Clin Endocrinol Metab 95: 2963-2968

8. DeFronzo RA (2004) Dysfunctional fat cells, lipotoxicity and type 2 diabetes. Int J Clin Pract 58(Suppl 143):9-21

9. Boden G (2003) Effects of free fatty acids (NEFA) on glucose metabolism: significance for insulin resistance and type 2 diabetes. Exp Clin Endocrinol Diabetes 111:121-124

10. Boden G, Lebed B, Schatz M, Homko C, Lemieux S (2001) Effects of acute changes of plasma free fatty acids on intramyocellular fat content and insulin resistance in healthy participants. Diabetes 50: 1612-1617

11. Roden M, Price TB, Perseghin G et al (1996) Mechanism of free fatty acid-induced insulin resistance in humans. J Clin Invest 97:28592865

12. Chavez AO, Kamath S, Jani R et al (2010) Effect of short-term free fatty acids elevation on mitochondrial function in skeletal muscle of healthy individuals. J Clin Endocrinol Metab 95:422-429

13. Yoshino J, Almeda-Valdes P, Patterson BW et al (2014) Diurnal variation in insulin sensitivity of glucose metabolism is associated with diurnal variations in whole-body and cellular fatty acid metabolism in metabolically normal women. J Clin Endocrinol Metab 99:E1666E1670

14. Schlierf G, Dorow E (1973) Diurnal patterns of triglycerides, free fatty acids, blood sugar, and insulin during carbohydrate-induction in man and their modification by nocturnal suppression of lipolysis. J Clin Invest 52:732-740

15. Spiegel K, Leproult R, Colecchia E et al (2000) Adaptation of the 24hour growth hormone profile to a state of sleep debt. Am J Physiol 279:R874-R883

16. Leproult R, Copinschi G, Buxton O, van Cauter E (1997) Sleep loss results in an elevation of cortisol levels the next evening. Sleep 20: $865-870$
17. Omisade A, Buxton OM, Rusak B (2010) Impact of acute sleep restriction on cortisol and leptin levels in young women. Physiol Behav 99:651-656

18. Iber C, Ancoli-Israel S, Chesson A, Quan S (2007) The AASM manual for the scoring of sleep and associated events: rules, terminology and technical specification. American Academy of Sleep Medicine, Westchester

19. Van Cauter E (1981) Quantitative methods for the analysis of circadian and episodic hormone fluctuations. In: van Cauter E, Copinschi $\mathrm{G}$ (eds) Human pituitary hormones: circadian and episodic variations. Martinus Nyhoff, the Hague, pp 1-25

20. Rosenthal MJ, Woodside WF (1988) Nocturnal regulation of free fatty acids in healthy young and elderly men. Metabolism 37:645-648

21. Clore JN, Nestler JE, Blackard WG (1989) Sleep-associated fall in glucose disposal and hepatic glucose output in normal humans. Putative signaling mechanism linking peripheral and hepatic events. Diabetes 38:285-290

22. Schlierf G, Reinheimer W, Stossberg V (1971) Diurnal patterns of plasma triglycerides and free fatty acids in normal participants and in patients with endogenous (type IV) hyperlipoproteinemia. Nutr Metab 13:80-91

23. Roust LR, Jensen MD (1993) Postprandial free fatty acid kinetics are abnormal in upper body obesity. Diabetes 42:1567-1573

24. Nedeltcheva AV, Imperial JG, Penev PD (2012) Effects of sleep restriction on glucose control and insulin secretion during diet-induced weight loss. Obesity (Silver Spring) 20:1379-1386

25. Wehrens SM, Hampton SM, Finn RE, Skene DJ (2010) Effect of total sleep deprivation on postprandial metabolic and insulin responses in shift workers and non-shift workers. J Endocrinol 206:205-215

26. Rasch B, Dodt C, Molle M, Born J (2007) Sleep-stagespecific regulation of plasma catecholamine concentration. Psychoneuroendocrinology 32:884-891

27. Carter JR, Durocher JJ, Larson RA, DellaValla JP, Yang H (2012) Sympathetic neural responses to 24-hour sleep deprivation in humans: sex differences. Am J Physiol Heart Circ Physiol 302: H1991-H1997

28. Chapotot F, Buguet A, Gronfier C, Brandenberger G (2001) Hypothalamo-pituitary-adrenal axis activity is related to the level of central arousal: effect of sleep deprivation on the association of highfrequency waking electroencephalogram with cortisol release. Neuroendocrinology 73:312-321

29. Scheer FA, Hilton MF, Mantzoros CS, Shea SA (2009) Adverse metabolic and cardiovascular consequences of circadian misalignment. Proc Natl Acad Sci U S A 106:4453-4458

30. Boden G, Chen X, Urbain JL (1996) Evidence for a circadian rhythm of insulin sensitivity in patients with NIDDM caused by cyclic changes in hepatic glucose production. Diabetes 45:1044-1050

31. Kashyap SR, Belfort R, Berria R et al (2004) Discordant effects of a chronic physiological increase in plasma NEFA on insulin signaling in healthy participants with or without a family history of type 2 diabetes. Am J Physiol Endocrinol Metab 287:E537-E546

32. Kim SP, Catalano KJ, Hsu IR, Chiu JD, Richey JM, Bergman RN (2007) Nocturnal free fatty acids are uniquely elevated in the longitudinal development of diet-induced insulin resistance and hyperinsulinemia. Am J Physiol Endocrinol Metab 292:E1590-E1598

33. Wright KP Jr, Hull JT, Hughes RJ, Ronda JM, Czeisler CA (2006) Sleep and wakefulness out of phase with internal biological time impairs learning in humans. J Cogn Neurosci 18:508-521

34. Markwald RR, Melanson EL, Smith MR et al (2013) Impact of insufficient sleep on total daily energy expenditure, food intake, and weight gain. Proc Natl Acad Sci U S A 110:5695-5700

35. Maury E, Hong HK, Bass J (2014) Circadian disruption in the pathogenesis of metabolic syndrome. Diabetes Metab 40:338-346

36. Shostak A, Meyer-Kovac J, Oster H (2013) Circadian regulation of lipid mobilization in white adipose tissues. Diabetes 62:2195-2203 